\title{
Serotypes associated with the development of pneumococcal para-pneumonic effusion in adults
}

\author{
Thomas Bewick¹, Carmen Sheppard², Sonia Greenwood', Mary Slack², \\ Caroline Trotter ${ }^{3}$, Robert George ${ }^{2}$ and Wei Shen Lim¹
}

Affiliations: 'Dept of Respiratory Medicine, Nottingham University Hospitals NHS Trust, Nottingham, ${ }^{2}$ Respiratory and Systemic Infection Laboratory, Microbiology Services Division, Health Protection Agency, London, and ${ }^{3}$ School of Social and Community Medicine, University of Bristol, Bristol, UK.

Correspondence: T. Bewick, Dept of Respiratory Medicine, Nottingham University Hospitals NHS Trust, Nottingham, NG5 1PB, UK. E-mail: thomasbewick@doctors.org.uk

ABSTRACT Serotypes 1, 3, 7F and 19A are implicated in childhood pneumococcal para-pneumonic effusion (PPE). It is not known whether the same is true for adult PPE.

A prospective cohort study was conducted over a 2-year period. Consecutive adults admitted with community-acquired pneumonia (CAP) were studied. Pneumococcal serotype was identified from urine samples using a multiplex immunoassay.

Of 920 patients recruited, 366 had pneumococcal CAP; 100 of these had PPE and a serotype was determined in 73 patients. Factors associated with PPE were age, pneumonia severity index score and serotype. Serotypes most associated with PPE were 1 (18 (45\%) out of 40), 19A (9 (45\%) out of 20) and 3 (8 $(40 \%)$ out of 20$)$. Serotypes common in childhood PPE were independently associated with adult PPE (adjusted OR 2.3; $\mathrm{p}=0.003$ ). Serotypes not included in the 7 -valent pneumococcal conjugate vaccine $(\mathrm{PCV})$ were more likely to be associated with PPE (OR 2.1; $\mathrm{p}=0.024)$ compared with those in the vaccine. Serotypes included in PCV-13 were as likely to be associated with PPE as those that are not (OR 0.8; $\mathrm{p}=0.301)$.

Serotypes 1, 3, 7F and 19A are independently associated with adult PPE, a similar finding to childhood PPE. Serotype replacement following pneumococcal vaccine implementation may influence the spectrum of clinical disease.

@ERSpublications

Serotypes associated with pneumococcal para-pneumonic effusion in adults are similar to those in childhood disease http://ow.ly/10nxw

Received: Sept 122012 | Accepted after revision: Nov 252012 | First published online: Dec 202012

Support statement: This work was supported by an unrestricted grant from Pfizer. The study was conceived by the authors and the study design was developed and agreed by the authors without any input from the funding body. The study concept and design was peer reviewed by an independent reviewer prior to ethics approval and prior to award of the grant from the funding body. The funding body was not involved in, nor had any influence over, study design, data collection, data analyses, interpretation of results, report writing, or in the decision to submit the paper for publication. All study data are held solely by the authors. The sponsor for the study was Nottingham University Hospitals NHS Trust. The authors are grateful to Alere (Stockport, UK; formerly Inverness Medical) for manufacturing and providing Binax NOW ${ }_{\circledast}$ test kits.

Conflict of interest: Disclosures can be found alongside the online version of this article at www.erj.ersjournals.com 


\section{Introduction}

Para-pneumonic effusions (PPEs) complicate community-acquired pneumonia (CAP) in adults in $\geqslant 10 \%$ of cases [1-3], and are associated with poorer outcome [4]. There is substantial variation in the incidence of PPE by causative organism, with the incidence lower in Mycoplasma pneumoniae and viral infection [5], but higher when the cause is Streptococcus pneumoniae [6]. The commonest cause of CAP, S. pneumoniae [7, 8], may be divided into over 90 different serotypes [9], many of which show distinctly different clinical features in both adults and children. Serotypes 1,3,19A and 7F have been particularly associated with development of pneumococcal PPE and empyema in children [10-12], but no such association has been reported to date in adults. Although no risk factors have been specifically identified for the development of pneumococcal PPE, factors associated with the development of complicated pneumococcal CAP (defined as multilobar CAP, PPE or empyema) include chronic liver disease, high C-reactive protein (CRP) levels on admission and creatinine level, with chronic obstructive pulmonary disease (COPD) a negative risk factor [13]. Risk factors for all-cause complicated PPE include age $\geqslant 60$ years, alcoholism, pleuritic chest pain, tachycardia, leukocytosis, low albumin, low plasma sodium and high platelet count $[14,15]$.

Seven-, 10-, and 13-valent pneumococcal conjugate vaccines (PCVs) have been licensed in recent years for use in preventing pneumococcal disease in children (with nine- and 11-valent vaccines trialled and not licensed, and a 15-valent vaccine under development). The seven-valent conjugate vaccine (PCV-7) was added to UK childhood immunisation schedules in September 2006, and was replaced by a 13-valent vaccine (PCV-13) in April 2010. Following the introduction of the conjugate vaccines to infant immunisation schedules a shift was observed in the serotypes seen in adult invasive pneumococcal disease towards nonvaccine-type serotypes [16, 17]. Some studies have reported a relative rise in the incidence of all-cause and pneumococcal PPE in children following the introduction of PCV-7 [11, 18-20], as the serotypes commonly associated with pneumococcal PPE are not included in PCV-7, although in the UK a fall in paediatric empyema hospitalisations was observed [21]. It is too early to say whether there has been a subsequent decrease in the incidence of pneumococcal PPE in children following the introduction of PCV13 (which includes serotypes $1,3,5,6 \mathrm{~A}, 7 \mathrm{~F}$ and 19A) due to continued serotype replacement with nonPCV-13 serotypes. Monitoring of pneumococcal serotypes and their associated clinical patterns of disease is therefore important and has not been performed for pneumococcal PPE in adults to date.

The aims of this paper were 1) to describe the serotype distribution of pneumococcal PPE in adult noninvasive CAP, and 2) to ascertain whether serotypes prevalent in childhood PPE are also implicated in adult PPE, independent of potential confounding variables.

\section{Methods}

Study design

The study design and recruitment methods are described elsewhere, and participants in this study were drawn from an earlier publication [22]. This study is novel in that it presents a detailed analysis of the association between the different pneumococcal serotypes previously described and the occurrence of simple and complicated PPEs. Briefly, consecutive adult patients (aged $\geqslant 16$ years) admitted between September 2008 and September 2010 with CAP to two large UK teaching hospitals were prospectively recruited as part of an observational cohort study. Patients were included if they had at least one acute symptom consistent with a lower respiratory tract infection (breathlessness, cough, sputum or fever), had new infiltrates on chest radiograph, and were treated by the admitting team for CAP. Patients were excluded if they had been admitted to hospital in the preceding 10 days, had tuberculosis or had post-obstructive pneumonia due to lung cancer.

Urine samples were obtained from each patient at the start of the admission episode. These were tested using the Binax NOW $\mathrm{N}_{\circledast}$ immunochromatographic test kit (Alere Ltd, Stockport, UK) and tested in batches by a

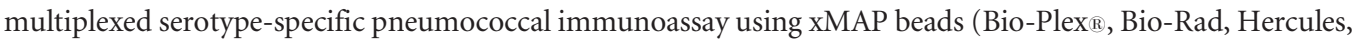
CA, USA). The assay detects 14 pneumococcal serotypes $(1,3,4,5,6 \mathrm{~A} / \mathrm{C}, 6 \mathrm{~B}, 7 \mathrm{~F} / \mathrm{A}, 8,9 \mathrm{~V}, 14,18,19 \mathrm{~A}, 19 \mathrm{~F}$ and $23 \mathrm{~F}$ ) with a sensitivity of $79 \%$ and specificity of $99 \%$ [23]. Serotype was also determined in bacteraemic pneumococcal patients by means of slide agglutination with latex pool sera and standard group and factor sera (Statens Serum Institut, Copenhagen, Denmark) at the Respiratory and Systemic Infection Laboratory, Health Protection Agency Microbiology Services Division (London, UK). Routine blood, pleural fluid and sputum samples for bacteriology culture were collected by admitting teams according to local CAP guidelines and processed in the Nottingham University Hospitals Department of Clinical Microbiology (Nottingham, UK) as part of standard clinical care. Culture results were recorded by the study investigators.

\section{Definitions}

PPE is defined as a patient meeting the inclusion criteria for CAP as above, with a new effusion on chest radiograph as recorded by the reporting radiologist. This definition concurs with the British Thoracic 
Society pleural guidelines [24], and can represent a minimum of $\sim 200 \mathrm{~mL}$ of pleural fluid [25]. Pleural ultrasound was not performed on every patient unless requested and performed by the relevant clinical team. Complicated PPE is defined as any effusion requiring intercostal chest drainage (including surgical therapy if tube drainage was not deemed possible).

Patients were defined as having pneumococcal CAP if any microbiological test was positive for $S$. pneumoniae, including blood, pleural fluid or sputum culture, Binax NOW ${ }_{\circledast}$ or Bio-Plex serotype-specific antigen detection. Patients with pneumococcal CAP where no serotype was detected after testing with the Bio-Plex assay (i.e. S. pneumoniae detected by Binax NOW ${ }_{\circledR}$ or sputum culture, but no serotype determined by Bio-Plex or blood culture) were described as having "untyped" pneumococcal CAP. Serotypes included in PCV-7 (4, 6B, 9V, 14, 18C, 19F and 23F) are hereafter referred to as "PCV-7 vaccine-type (VT)" and serotypes not contained within PCV-7 are referred to as "non-vaccine-type" (NVT). Serotypes included in PCV-13 (1, 3, 4, 5, 6A, 6B, 7F, 9V, 14, 18C, 19A, 19F and 23F) are hereafter referred to as "PCV-13 VT". Serotypes associated with PPE in paediatric cohorts $(1,3,7 \mathrm{~F}$ and 19A) are defined as "PPE-related serotypes". All PCV-7 and PCV-13 VT serotypes are detectable by the Bio-Plex assay. Highly invasive serotypes were defined as 1, 5, 7F and 8 according to previous publications [22], with the remainder defined as less invasive serotypes.

\section{Analysis}

Statistical calculations were made using SPSS v16.0 (IBM SPSS, Armonk, NY, USA). Categorical data were compared using Pearson's Chi-squared test, which was also used for calculating odds ratios and 95\% confidence intervals. Continuous data that were non-normally distributed were compared using the MannWhitney U-test. Differences in 30-day mortality, pneumonia severity (as measured by the pneumonia severity index (PSI)) [3] and length of hospital stay (LOS) were investigated between patients with PPE and patients with CAP in the absence of PPE. Within the subgroup of patients with pneumococcal CAP, associations were investigated between CAP caused by PPE-related serotypes and the development of PPE, and which (if any) individual serotypes had a predisposition to causing PPE.

To adjust for potential confounding variables, we performed a multivariable logistic regression analysis. Although no previous publications have identified risk factors for pneumococcal PPE, we inferred potential risk factors from studies identifying risk factors for complicated PPE and complicated CAP (defined as multilobar CAP or CAP with PPE or empyema) [13-15]. These include age $<60$ years, tachycardia $>100$ beats $\cdot \mathrm{min}^{-1}$, leukocytosis $>15000 \mathrm{~mm}^{-3}$, CRP $>100 \mathrm{mg} \cdot \mathrm{L}^{-1}$, presence of chronic liver disease or COPD, serum creatinine $>130 \mu \mathrm{mol} \mathrm{L} \mathrm{L}^{-1}$, sodium $<130 \mathrm{mmol} \cdot \mathrm{L}^{-1}$ and platelet count $>400 \times 10^{9} \cdot \mathrm{L}^{-1}$. (Note that pleuritic pain, albumin $<30 \mathrm{~g} \cdot \mathrm{L}^{-1}$ and alcohol abuse, although identified as potential risk factors for complicated PPE, were not recorded in all patients and therefore not included in the analysis.) Analyses were also performed to adjust for severity of CAP using PSI risk group [3].

\section{Results}

Of 1100 patients identified with CAP during the study period, 956 consented to be included in the study. 36 patients were unable to provide a urine sample (and had no other test positive for pneumococcus), leaving 920 for analysis. The baseline demographics and clinical characteristics of the study population are described in table 1. Of the 920 participants enrolled in the study, 366 were found to have pneumococcal aetiology. Of these, 100 (27.3\%) were associated with PPE at presentation compared with 109 (19.7\%) out of 554 patients with nonpneumococcal aetiology (OR 1.5, 95\% CI 1.1-2.1; $p=0.007$ ). This association was maintained after adjustment for disease severity (OR 1.5, 95\% CI 1.1-2.1; $\mathrm{p}=0.010$ ). Of 209 patients with all-cause PPE, 56 (26.8\%) had complicated PPE; comprising of 30 (30\%) out of 100 patients with pneumococcal PPE compared with 26 (23.9\%) out of 109 patients with non-pneumococcal PPE (OR 1.8, 95\% CI 1.1-3.1; $\mathrm{p}=0.029)$. Thoracocentesis was performed in 83 (39.7\%) out of 209 PPEs; pleural fluid culture was positive for pneumococcus in two patients.

\section{PPE and outcome}

All-cause PPE was associated with higher 30-day mortality compared with patients without PPE (29 (13.9\%) out of 209 versus $63(8.9 \%)$ out of 711 ; OR $1.7,95 \%$ CI $1.0-2.7 ; \mathrm{p}=0.034)$ on univariate analysis, but not after adjustment for disease severity (OR 1.4, 95\% CI $0.9-2.3 ; \mathrm{p}=0.15$ ). The incidence of PPE increased with increasing pneumonia severity (PSI classes I-III: 68 (17.2\%) out of 395; PSI class IV: 89 $(26.5 \%)$ out of 336; PSI class V: $52(27.5 \%)$ out of 189 ; $\mathrm{p}$ value for trend=0.002). Median (interquartile range) LOS in those who survived to discharge $(n=832)$ was longer for patients with PPE (10 (5-17) days, versus 6 (4-11) days; $\mathrm{p}<0.001$ ). 
TABLE 1 Characteristics and outcomes of the study cohort presenting with community-acquired pneumonia (all cause)

\begin{tabular}{|c|c|c|c|c|c|}
\hline & Whole cohort & PPE & No PPE & Complicated PPE & p-value $\#$ \\
\hline Subjects & 920 & 209 & 711 & 56 & \\
\hline \multicolumn{6}{|l|}{ Demographics } \\
\hline Male & 546 (59.3) & $116(55.5)$ & $430(60.5)$ & 33 (58.9) & 0.198 \\
\hline $\begin{array}{l}\text { Residential or nursing care home } \\
\text { resident }\end{array}$ & $51(5.5)$ & $8(3.8)$ & $43(6.0)$ & $1(1.8)$ & 0.222 \\
\hline Ischaemic heart disease & $145(15.8)$ & $28(13.4)$ & $118(16.6)$ & 7 (12.5) & 0.625 \\
\hline Diabetes mellitus & $130(14.1)$ & $35(16.7)$ & $95(13.4)$ & 8 (14.3) & 0.200 \\
\hline Cerebrovascular disease & $103(11.2)$ & $26(12.4)$ & $77(10.8)$ & $6(10.7)$ & 0.506 \\
\hline Asthma & $102(11.1)$ & $28(13.4)$ & $74(10.4)$ & $5(8.9)$ & 0.226 \\
\hline Congestive cardiac failure & $74(8.0)$ & $20(9.6)$ & $54(7.6)$ & $4(7.1)$ & 0.349 \\
\hline Active malignancy & $67(7.3)$ & $13(6.2)$ & $54(7.6)$ & $4(7.1)$ & 0.509 \\
\hline PPV in preceding 10 years & $383 / 824$ (46.5) & $85 / 191(44.5)$ & $298 / 633(47.1)$ & $14 / 53(26.4)$ & 0.532 \\
\hline Severity & & & & & 0.002 \\
\hline PSI class I-III & $395(42.9)$ & 68 (32.5) & $327(46.0)$ & $31(55.4)$ & \\
\hline PSI class IV & $336(36.5)$ & $89(42.6)$ & 247 (34.7) & $18(32.1)$ & \\
\hline PSI class V & $189(20.5)$ & 52 (24.9) & 137 (19.3) & 7 (12.5) & \\
\hline Aetiology & & & & & 0.007 \\
\hline Pneumococcal & 366 (39.8) & $100(47.8)$ & $266(37.4)$ & $30(53.6)$ & \\
\hline Nonpneumococcal & $554(60.2)$ & 109 (52.2) & $445(62.6)$ & $26(46.4)$ & \\
\hline \multicolumn{6}{|l|}{ Outcome } \\
\hline 30-day mortality & $92(10.0)$ & 29 (13.9) & 63 (8.9) & $4(7.1)$ & 0.034 \\
\hline LOS days & $7(4-12)$ & $10(5-17)$ & $6(4-11)$ & $13(5-15)$ & $<0.001$ \\
\hline IRVS & $82(8.9)$ & $23(11.0)$ & $59(8.3)$ & $5(8.9)$ & 0.227 \\
\hline
\end{tabular}

Data are presented as $n$, median (interquartile range), $n(\%)$ or $n / N(\%)$, unless otherwise stated. PPE: para-pneumonic effusion; WHO: World Health Organization; COPD: chronic obstructive pulmonary disease; PPV: adult pneumococcal polysaccharide vaccine; PSI: pneumonia severity index; LOS: length of hospital stay; IRVS: need for intensive respiratory or vasopressor support. \#: p-value compares patients with PPE to those without PPE.

\section{Serotypes in adult pneumococcal PPE}

A serotype was determined in $246(67.2 \%)$ out of 366 patients with pneumococcal CAP, including 40 patients with a positive blood culture. The prevalence of PPE was highest for patients with serotypes 19A (9 $(45 \%)$ out of 20$), 1(18(45 \%)$ out of 40$)$ and $3(8(40 \%)$ out of 20$)$ (table 2$)$. Of 94 adults with a serotype identified as likely to cause pneumococcal PPE from paediatric studies (serotypes 1, 3, 7F and 19A), 38 (40.4\%) had PPE, compared to 35 (23.0\%) out of 152 with other serotypes (unadjusted OR 2.3, 95\% CI $1.3-4.0 ; \mathrm{p}=0.004)$ and $27(22.5 \%)$ out of 120 with untyped pneumococcal CAP (OR 2.3, 95\% CI 1.3-4.2; $\mathrm{p}=0.007$ ) (fig. 1 and table 3). After adjustment for all putative risk factors, CAP due to PPE-related serotypes remained significantly associated with PPE when compared to other pneumococcal cases (OR 2.3, 95\% CI 1.3-4.1; $\mathrm{p}=0.003$ ), or the untyped group alone (OR 2.5, 95\% CI 1.2-4.8; $\mathrm{p}=0.007$ ) (table 4).

Of the PPE-related serotypes, serotypes 1 and 19A were individually associated with PPE when compared with the untyped group (serotype 1: adjusted OR 2.8, 95\% CI 1.3-6.0; $\mathrm{p}=0.007$; serotype 19A: adjusted OR 2.8, 95\% CI 1.1-7.5; $\mathrm{p}=0.038)$. These associations were maintained when adjusted for disease severity (serotype 1: adjusted OR 3.1, 95\% CI 1.5-6.8; $\mathrm{p}=0.004$; serotype 19A: adjusted OR 3.1, 95\% CI 1.1-8.2; $\mathrm{p}=0.027$ ) or adjusted for putative risk factors for pneumococcal PPE (serotype 1: adjusted OR 2.4, 95\% CI 1.1-5.5; $\mathrm{p}=0.034$; serotype 19A: adjusted OR 2.7, 95\% CI 1.0-7.7; $\mathrm{p}=0.061)$. Only six serotypes occurred in cases of complicated pneumococcal PPE: $1(\mathrm{n}=9), 19 \mathrm{~A}(\mathrm{n}=4)$, and 3, 4, 8 and $14($ all $\mathrm{n}=2)$.

Patients with a pneumococcal serotype not included within PCV-7 $(n=170)$ were more likely to present with PPE compared with those with a PCV-7 serotype (58 (34.1\%) out of 170 versus 14 (19.4\%) out of 72; OR 2.1, 95\% CI 1.1-4.2; $\mathrm{p}=0.024)$. There was no evidence for a difference in the odds of PPE in patients 
TABLE 2 Proportion of cases caused by each serotype by disease site

\begin{tabular}{|c|c|c|c|c|}
\hline Serotype & All pneumococcal & Pneumococcal PPE & Complicated pneumococcal PPE & Multilobar \\
\hline 3 & 20 & $8(40)$ & $2(10)$ & 7 (35) \\
\hline 5 & 18 & 6 (33) & $0(0)$ & 6 (33) \\
\hline 6A/C & 11 & $1(9)$ & $0(0)$ & $4(36)$ \\
\hline 6B & 2 & $0(0)$ & $0(0)$ & $1(50)$ \\
\hline 9V & 4 & $1(25)$ & $0(0)$ & $1(25)$ \\
\hline 14 & 45 & $8(18)$ & $2(4)$ & $20(44)$ \\
\hline $18 C$ & 4 & $0(0)$ & $0(0)$ & $0(0)$ \\
\hline 19A & 20 & $9(45)$ & $4(20)$ & 7 (35) \\
\hline $19 \mathrm{~F}$ & 3 & $1(33)$ & $0(0)$ & 1 (33) \\
\hline $23 \mathrm{~F}$ & 0 & $0(0)$ & $0(0)$ & $0(0)$ \\
\hline
\end{tabular}

Data are presented as $\mathrm{n}$ or $\mathrm{n}(\%)$. PPE: para-pneumonic effusion.

with PCV-13 VT serotype compared to a combined group of those with a serotype not included in PCV-13 or with untyped pneumococcal CAP $(\mathrm{n}=155$; OR $0.8,95 \%$ CI $0.5-1.2 ; \mathrm{p}=0.301)$. Highly invasive serotypes $(1,5,7 \mathrm{~F}$ and 8$)$ were positively associated with PPE (38 (35.6\%) out of 107 versus 62 (23.9\%) out of 259; OR $1.8,95 \%$ CI 1.1-2.8; $\mathrm{p}=0.024)$, but not with complicated PPE $(12(31.6 \%)$ out of 38 versus $18(29.0 \%)$ out of 62 ; OR $1.1,95 \%$ CI $0.5-2.7 ; \mathrm{p}=0.787$ ).

\section{Discussion}

The main findings from this study are that PPE and complicated PPE are strongly associated with pneumococcal aetiology, and the serotypes independently associated with adult PPE are similar to those seen in childhood PPE, namely serotypes 1, 3, 7F and 19A. In particular, serotypes 1 and 19A are strongly associated with adult PPE. Pneumococcal serotype can have profound implications for the spectrum of clinical disease, and has been shown to influence 30-day mortality, invasive disease potential and disease severity $[26,27]$. To our knowledge, this is the only study in adults with CAP to investigate the relationship between pneumococcal serotype and both invasive and noninvasive pneumococcal PPE.

FIGURE 1 Proportion of each pneumococcal serotype group with para-pneumonic effusion (PPE). PPE-associated serotypes include $1,3,7 \mathrm{~F}$ and 19A.

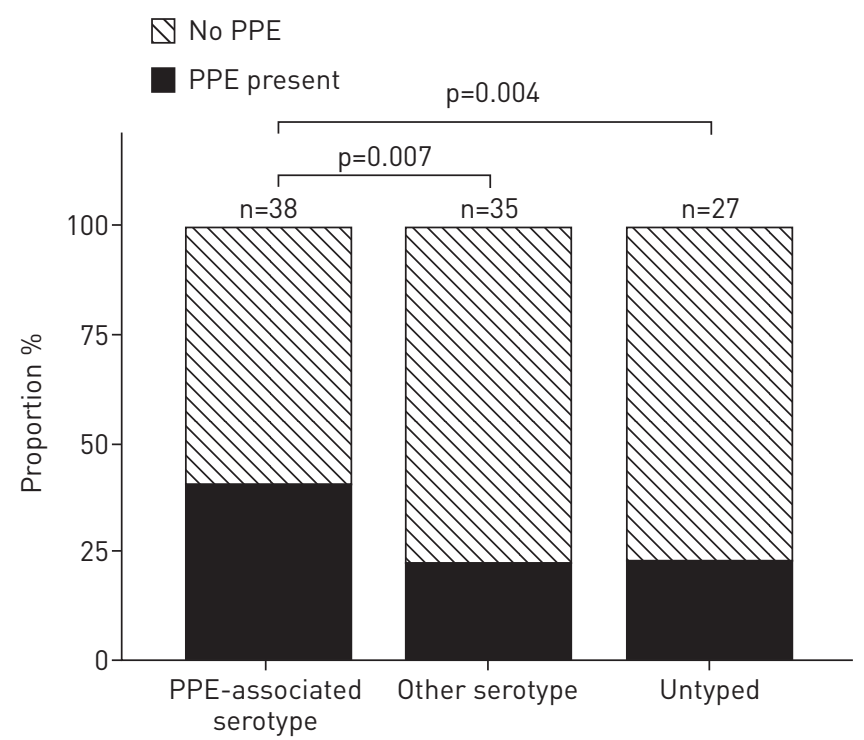


TABLE 3 Comparison of clinical characteristics and outcomes of patients with pneumococcal community-acquired pneumonia (CAP)

No PPE

Pneumococcal CAP

PPE

\begin{tabular}{|c|c|c|}
\hline $\begin{array}{l}\text { PPE-associated } \\
\text { serotypes }^{\#}\end{array}$ & Other serotypes & OR $(95 \% \text { CI })^{\top}$ \\
\hline
\end{tabular}

p-value

\begin{tabular}{|c|c|c|c|c|c|}
\hline \multicolumn{6}{|l|}{$\begin{array}{l}\text { Clinical feature at } \\
\text { presentation }\end{array}$} \\
\hline Age years & $72.2(57.9-82.1)$ & $65.0(42.0-78.7)$ & $65.3(47.6-78.7)$ & $0.99(0.98-1.02)$ & 0.900 \\
\hline COPD & $70(26.3)$ & $10(26.3)$ & $14(22.6)$ & $1.22(0.48-3.12)$ & 0.671 \\
\hline Asthma & $32(12.0)$ & $3(7.9)$ & $12(19.4)$ & $0.36(0.09-1.36)$ & 0.119 \\
\hline Creatinine $\mu \mathrm{mol} \cdot \mathrm{L}^{-1}$ & $101(75-143)$ & $90(76-131)$ & $93(70-159)$ & $1.00(0.99-1.00)$ & 0.319 \\
\hline Bacteraemia & 28 (10.5) & $8(21.1)$ & $4(6.5)$ & 3.87 (1.08-13.89) & 0.029 \\
\hline Shock $^{+}$ & $32(12.0)$ & $6(15.8)$ & 6 (9.7) & $1.12(0.36-3.44)$ & 0.845 \\
\hline \multicolumn{6}{|l|}{ PSI class } \\
\hline$|-|||$ & $117(44.0)$ & 12 (31.6) & 22 (35.5) & & \\
\hline IV & $92(34.6)$ & $17(44.7)$ & $23(37.1)$ & & 0.750 \\
\hline V & $57(21.4)$ & $9(23.7)$ & $17(27.4)$ & & \\
\hline
\end{tabular}

In the only other related study in adults, serotypes were only available in patients who had invasive pneumococcal disease $(n=84)$ [13]. That study identified serotypes 1,3 and $19 \mathrm{~A}$ as being most frequently found in "complicated CAP", which included multilobar disease as well as PPE and empyema. Paediatric PPE increased in incidence following PCV-7 introduction and prior to the introduction of PCV-13 [11, 1820], with PCV-7 NVT serotypes 1, 3, 5, 7F and 19A implicated [10-12]. In a small study of paediatric empyema, pneumococci were identified in 13 samples, six of which were serotype 19A and one serotype 1, the others being serotypes $14(n=1), 34(n=1)$ and untypable $(n=4)$ [28]. In another study, serotype 1 was particularly associated with complicated CAP (defined as PPE, empyema or necrotising CAP) in children

TABLE 4 Multivariate logistic regression analysis of hypothesised risk factors for pneumococcal para-pneumonic effusion (PPE), with untyped pneumococcal communityacquired pneumonia (CAP) as a reference group

\begin{tabular}{|c|c|c|}
\hline & OR $(95 \% \mathrm{CI})$ & p-value \\
\hline Age $<60$ years & $2.7(1.5-4.9)$ & 0.001 \\
\hline PPE-related serotype ${ }^{\#}$ & $2.5(1.3-4.8)$ & 0.007 \\
\hline Other serotype & $1.1(0.6-2.0)$ & 0.766 \\
\hline Creatinine $>130 \mu \mathrm{mol} \cdot \mathrm{L}^{-1}$ & $1.1(0.6-2.0)$ & 0.674 \\
\hline $\mathrm{CRP}>100 \mathrm{mg} \cdot \mathrm{L}^{-1}$ & $1.0(0.5-1.7)$ & 0.897 \\
\hline COPD & $1.6(0.8-3.0)$ & 0.189 \\
\hline Chronic liver disease & $0.7(0.1-3.7)$ & 0.695 \\
\hline Sodium $<130 \mathrm{mmol} \cdot \mathrm{L}^{-1}$ & $1.7(0.8-3.6)$ & 0.141 \\
\hline Platelets $>400 \times 10^{9} \mathrm{~L}^{-1}$ & $1.9(0.7-4.6)$ & 0.207 \\
\hline Tachycardia $>100$ beats $\cdot \mathrm{min}^{-1}$ & $0.5(0.3-0.9)$ & 0.012 \\
\hline Leukocytes $>15000$ cells $\cdot \mathrm{mm}^{-3}$ & $0.7(0.4-1.2)$ & 0.160 \\
\hline
\end{tabular}

Bold type represents significance at $p<0.05$. CRP: C-reactive protein; COPD: chronic obstructive pulmonary disease. " ${ }^{\#}$ 1, 3, 7F and $19 \mathrm{~A}^{\circ}{ }^{\circ}$ : all other detectable serotypes, excluding the untyped group. 
when compared with other serotypes [29]. No data are yet available on the serotype distribution of PPE since PCV-13 was introduced in 2010.

The serotypes identified as associated with PPE and complicated PPE in this study are similar to the findings of studies conducted in infants and children $[11,12]$. This raises the suggestion that for serotypes 1, 3, 7F and 19A, pathogen-related factors may be more important in the pathogenesis of PPE compared with age or host-related factors. The mechanisms whereby these serotypes might cause PPE are unclear. Zwitterionic polysaccharides contained within the serotype 1 coat can directly activate T-helper cells in a similar way to proteins via a major histocompatibility complex class II-dependent pathway [30]. In addition, it has been suggested that serotypes with a lower degree of encapsulation have a higher degree of interaction with the respiratory epithelium, and hence cause invasive disease, of which PPE and empyema may be a manifestation [31]. Serotypes with a low degree of encapsulation include 1, 4, 5, 7F and 14, with 19A being of intermediate thickness. However, serotype 3 is heavily encapsulated, suggesting that there may be other significant interacting mechanisms underlying aetiology of PPE. Serotype also affects the amount of complement deposition and neutrophil phagocytosis [32], and adhesion to and invasion through the respiratory epithelium via variable exposure of bacterial proteins [33], although serotypes 1 and 19A have not been studied individually in this regard. More studies are required to explore fully this interaction between capsule type and host defence.

Of the previously described putative clinical risk factors for pneumococcal PPE, younger age was found to be independently associated with pneumococcal PPE. Interestingly, pleuritic chest pain is more commonly reported in younger patients with pneumococcal CAP than in older patients [34], and has previously been associated with the development of PPE [15]. The observed increased incidence of PPE and infection with PPE-related serotypes in younger patients provides a potential explanation for this finding, although the underlying mechanisms are unclear. Tachycardia was unexpectedly found to be negatively associated with PPE in the cohort with pneumococcal CAP, although it was not associated with all-cause PPE. In contrast, FALGUERA et al. [15] reported an independent association of tachycardia $>100$ beats $\cdot \mathrm{min}^{-1}$ with all-cause empyema and complicated PPE.

The outcomes of patients with PPE have consistently been shown to be worse than those without PPE, and this study confirms these findings. In particular, length of stay is significantly longer for patients with PPE. Effusions requiring chest tube drainage would be expected to lengthen median length of stay, as would a higher proportion of more severe or more inflammatory disease. In addition, radiographic resolution of PPE may take longer than uncomplicated consolidation, thereby lengthening the duration of symptom recovery.

Following the introduction of conjugate vaccines to national childhood vaccination schedules, a fall in the incidence of invasive pneumococcal disease across all age groups, together with a significant change in the serotype distribution, has been observed [16]. In particular, serotypes 1 and 19A, along with other PCV-7 NVT serotypes increased in prevalence following the introduction of PCV-7 vaccination. The additional serotypes contained within PCV-13 $(1,3,5,6 \mathrm{~A}, 7 \mathrm{~F}$ and 19A) include those identified in this study as implicated in the development of pneumococcal PPE. Therefore, a potential unintended benefit of the introduction of PCV-13 may be a fall in the incidence of pneumococcal PPE over the next few years. However, further serotype replacement consequent on PCV-13 vaccination may mean that previously less common serotypes may emerge with an as yet unknown clinical impact. This underlines the importance of ongoing serotype surveillance, particularly for complicated CAP.

\section{Study limitations}

A number of limitations to the study have been discussed in a previous publication [22]. The main limitation of the current study is that a pleural fluid sample was not taken for microbiological analyses from all cases with a PPE. Thoracocentesis was performed at the discretion of the attending clinical team, and where not performed this is likely to be due to small size of the PPE and where chest drainage was not felt to be clinically imperative. This also limits the ability to differentiate the PPEs into their different stages. The assumption is made that the effusions present on chest radiograph are related to the concurrent consolidation, and are of the same aetiology and serotype. It would seem unlikely that a new effusion in combination with consolidation on chest radiograph would be unrelated, but we cannot exclude this possibility. Secondly, the putative risk factors for pneumococcal PPE that were examined in this study were based on two unvalidated studies; one identified risk factors for complicated pneumococcal CAP and the other all-cause complicated PPE. Therefore, there may be other potential confounders that might account for these findings.

\section{Conclusion}

Serotypes 1, 3, 7F and 19A are independently associated with adult PPE, a similar finding to childhood PPE. This suggests that pathogen factors are important in the development of PPE and that serotype replacement following pneumococcal vaccination strategies may impact on the spectrum of clinical disease. 


\section{Acknowledgements}

The authors thank Christine More and Seyi Eletu from Respiratory and Systemic Infection Laboratory (RSIL) Colindale (London, UK), who processed the urine specimens using the Bio-Plex assay, and Robert Cave, Adrian Patrick, Michelle Stannard, Joanne Palfreyman and Andrew Shelton from the Nottingham University Hospitals Microbiology Department (Nottingham, UK), who processed the urine specimens using the Binax NOW ${ }_{\circledR}$ assay. They are also grateful to Tim Harrison from RSIL Colindale for his support, and Gemma Thompson and Emily Jarvis (Nottingham University Hospitals NHS Trust, Nottingham, UK) for their help in recruiting participants. They thank the clinicians and nursing staff of Nottingham University Hospitals NHS Trust, in particular Melanie Caine, Gaynor Bates, and Jo-Anne Harvey, who helped to identify potential participants, and allowed the study investigators to see their patients.

\section{References}

Sahn SA. Diagnosis and management of parapneumonic effusions and empyema. Clin Infect Dis 2007; 45: 1480-1486.

2 Lim WS, van der Eerden MM, Laing R, et al. Defining community acquired pneumonia severity on presentation to hospital: an international derivation and validation study. Thorax 2003; 58: 377-382.

3 Fine MJ, Auble TE, Yealy DM, et al. A prediction rule to identify low-risk patients with community-acquired pneumonia. N Engl J Med 1997; 336: 243-250.

4 Hasley PB, Albaum MN, Li YH, et al. Do pulmonary radiographic findings at presentation predict mortality in patients with community-acquired pneumonia? Arch Intern Med 1996; 156: 2206-2212.

Fine NL, Smith LR, Sheedy PF. Frequency of pleural effusions in mycoplasma and viral pneumonias. N Engl J Med 1970; 283: 790-793.

6 Taryle DA, Potts DE, Sahn SA. The incidence and clinical correlates of parapneumonic effusions in pneumococcal pneumonia. Chest 1978; 74: 170-173.

7 Cillóniz C, Ewig S, Polverino E, et al. Microbial aetiology of community-acquired pneumonia and its relation to severity. Thorax 2011; 66: 340-346.

8 Lim WS, Macfarlane JT, Boswell TC, et al. Study of community acquired pneumonia aetiology (SCAPA) in adults admitted to hospital: implications for management guidelines. Thorax 2001; 56: 296-301.

9 Park IH, Pritchard DG, Cartee R, et al. Discovery of a new capsular serotype (6C) within serogroup 6 of Streptococcus pneumoniae. J Clin Microbiol 2007; 45: 1225-1233.

10 Spencer D, Thomas M, Mohammed E, et al. National surveillance of paediatric empyema in the UK; the UK-ESPE study. Eur Respir J 2012; 40: Suppl. 56, $2946 s$.

11 Byington CL, Hulten KG, Ampofo K, et al. Molecular epidemiology of pediatric pneumococcal empyema from 2001 to 2007 in Utah. J Clin Microbiol 2010; 48: 520-525.

$12 \mathrm{Yu}$ J, Salamon D, Marcon M, et al. Pneumococcal serotypes causing pneumonia with pleural effusion in pediatric patients. J Clin Microbiol 2011; 49: 534-538.

13 Cillóniz C, Ewig S, Polverino E, et al. Pulmonary complications of pneumococcal community-acquired pneumonia: incidence, predictors, and outcomes. Clin Microbiol Infect 2012; 18: 1134-1142.

14 Chalmers JD, Singanayagam A, Murray MP, et al. Risk factors for complicated parapneumonic effusion and empyema on presentation to hospital with community-acquired pneumonia. Thorax 2009; 64: 592-597.

15 Falguera $\mathrm{M}$, Carratalà $\mathrm{J}$, Bielsa $\mathrm{S}$, et al. Predictive factors, microbiology and outcome of patients with parapneumonic effusion. Eur Respir J 2011; 38: 1173-1179.

16 Miller E, Andrews NJ, Waight PA, et al. Herd immunity and serotype replacement 4 years after seven-valent pneumococcal conjugate vaccination in England and Wales: an observational cohort study. Lancet Infect Dis 2011; 11: 760-768.

17 Lexau CA, Lynfield R, Danila R, et al. Changing epidemiology of invasive pneumococcal disease among older adults in the era of pediatric pneumococcal conjugate vaccine. JAMA 2005; 294: 2043-2051.

18 Hendrickson DJ, Blumberg DA, Joad JP, et al. Five-fold increase in pediatric parapneumonic empyema since introduction of pneumococcal conjugate vaccine. Pediatr Infect Dis J 2008; 27: 1030-1032.

19 Grijalva CG, Zhu Y, Nuorti JP, et al. Emergence of parapneumonic empyema in the USA. Thorax 2011; 66: 663-668.

20 Li ST, Tancredi DJ. Empyema hospitalizations increased in US children despite pneumococcal conjugate vaccine. Pediatrics 2010; 125: 26-33.

21 Koshy E, Murray J, Bottle A, et al. Impact of the seven-valent pneumococcal conjugate vaccination (PCV7) programme on childhood hospital admissions for bacterial pneumonia and empyema in England: national timetrends study, 1997-2008. Thorax 2010; 65: 770-774.

22 Bewick T, Sheppard C, Greenwood S, et al. Serotype prevalence in adults hospitalised with pneumococcal noninvasive community-acquired pneumonia. Thorax 2012; 67: 540-545.

23 Sheppard CL, Harrison TG, Smith MD, et al. Development of a sensitive, multiplexed immunoassay using xMAP beads for detection of serotype-specific Streptococcus pneumoniae antigen in urine samples. J Med Microbiol 2011; 60: 49-55.

24 Maskell NA, Butland RJA. Pleural Diseases Group, Standards of Care Committee, British Thoracic Society. BTS guidelines for the investigation of a unilateral pleural effusion in adults. Thorax 2003; 58: Suppl. 2, ii8-ii17.

25 Blackmore CC, Black WC, Dallas RV, et al. Pleural fluid volume estimation: a chest radiograph prediction rule. Acad Radiol 1996; 3: 103-109.

26 Luján M, Gallego M, Belmonte $\mathrm{Y}$, et al. Influence of pneumococcal serotype group on outcome in adults with bacteraemic pneumonia. Eur Respir J 2010; 36: 1073-1079.

27 van Hoek AJ, Andrews N, Waight PA, et al. Effect of serotype on focus and mortality of invasive pneumococcal disease: coverage of different vaccines and insight into non-vaccine serotypes. PLoS One 2012; 7: e39150.

28 Lee JH, Kim SH, Lee J, et al. Diagnosis of pneumococcal empyema using immunochromatographic test on pleural fluid and serotype distribution in Korean children. Diagn Microbiol Infect Dis 2012; 72: 119-124.

29 Resti M, Moriondo M, Cortimiglia M, et al. Community-acquired bacteremic pneumococcal pneumonia in children: diagnosis and serotyping by real-time polymerase chain reaction using blood samples. Clin Infect Dis 2010; 51: 1042-1049. 
30 Velez CD, Lewis CJ, Kasper DL, et al. Type I Streptococcus pneumoniae carbohydrate utilizes a nitric oxide and MHC II-dependent pathway for antigen presentation. Immunology 2009; 127: 73-82.

31 Weinberger DM, Trzciński K, Lu YJ, et al. Pneumococcal capsular polysaccharide structure predicts serotype prevalence. PLoS Pathog 2009; 5: e1000476.

32 Hyams C, Camberlein E, Cohen JM, et al. The Streptococcus pneumoniae capsule inhibits complement activity and neutrophil phagocytosis by multiple mechanisms. Infect Immunol 2010; 78: 704-715.

33 Sanchez CJ, Hinojosa CA, Shivshankar P, et al. Changes in capsular serotype alter the surface exposure of pneumococcal adhesins and impact virulence. PLoS One 2011; 6: e26587.

34 Esposito AL. Community-acquired bacteremic pneumococcal pneumonia. Effect of age on manifestations and outcome. Arch Intern Med 1984; 144: 945-948. 